April 2005

\title{
The effects of greater occipital nerve block and trigger point injection on brush allodynia and pain in migraine
}

\author{
Avi Ashkenazi \\ Thomas Jefferson University \\ William B. Young \\ Thomas Jefferson University
}

Follow this and additional works at: https://jdc.jefferson.edu/neurologyfp

Part of the Neurology Commons

Let us know how access to this document benefits you

\section{Recommended Citation}

Ashkenazi, Avi and Young, William B., "The effects of greater occipital nerve block and trigger point injection on brush allodynia and pain in migraine" (2005). Department of Neurology Faculty Papers. Paper 9.

https://jdc.jefferson.edu/neurologyfp/9

This Article is brought to you for free and open access by the Jefferson Digital Commons. The Jefferson Digital Commons is a service of Thomas Jefferson University's Center for Teaching and Learning (CTL). The Commons is a showcase for Jefferson books and journals, peer-reviewed scholarly publications, unique historical collections from the University archives, and teaching tools. The Jefferson Digital Commons allows researchers and interested readers anywhere in the world to learn about and keep up to date with Jefferson scholarship. This article has been accepted for inclusion in Department of Neurology Faculty Papers by an authorized administrator of the Jefferson Digital Commons. For more information, please contact: JeffersonDigitalCommons@jefferson.edu. 
The Effects of Greater Occipital Nerve Block on Brush Allodynia and Pain in Migraine

\author{
Avi Ashkenazi, M.D. \\ Jefferson Headache Center \\ Department of Neurology \\ Thomas Jefferson University Hospital \\ Philadelphia, Pennsylvania \\ and \\ William B. Young, M.D. \\ Associate Professor, Department of Neurology and \\ Director of Inpatient Program, Jefferson Headache Center \\ Thomas Jefferson University Hospital \\ Philadelphia, Pennsylvania
}

Send reprints and correspondence to:

Avi Ashkenazi, M.D.

Jefferson Headache Center

Jefferson University Hospital

111 South 11th Street, STE 8130

Philadelphia, PA 19107

Tel. 215-955-2243; Fax. 215-955-2060

E-mail: avi.ashkenazi@jefferson.edu 


\begin{abstract}
Objective: To evaluate the effect of GONB, with or without trigger point injection (TPI), on dynamical mechanical (brush) allodynia (BA) and on head pain in migraine.

Background: Patients with migraine often have cutaneous allodynia, which is related to sensitization of central pain neurons. Greater occipital nerve block (GONB) is an effective treatment for migraine headache, however its effect on cutaneous allodynia in migraine is unknown.
\end{abstract}

Methods: We studied patients with migraine and BA who were treated with GONB with or without TPI. Demographic data, migraine history and headache features were documented. Allodynia was evaluated using a structured questionnaire and by applying a $4 x 4$-inch gauze pad to skin areas in the trigeminal and cervical dermatomes. Degree of allodynia (the allodynia score) was measured on a $100 \mathrm{~mm}$ visual analog scale (VAS) before treatment and 10 and 20 minutes thereafter. Headache levels were assessed using an 11-point verbal scale. Allodynia scores, as well as headache levels, before and after treatment were compared.

Results: Nineteen patients were studied. Mean age was $43.6 \pm 11.8$ years. Twenty minutes after treatment, headache was reduced in 17 patients $(89.5 \%)$ and did not change in two $(10.5 \%)$. The average headache level was 6.53 before treatment and 3.47 twenty minutes after it. The average allodynia score decreased after 20 minutes in all patients. Average allodynia score per site was reduced by $18.69 \mathrm{~mm}$ and $13.74 \mathrm{~mm}$ in the trigeminal and cervical areas, respectively. There was a positive correlation between allodynia index, obtained through the questionnaire, and allodynia score, obtained by examination. 
Conclusion: GONB, with or without TPI, reduced both head pain and brush allodynia in this migraine patient group.

Key words: greater occipital nerve block; migraine; allodynia

\author{
Abbreviations: \\ GONB - greater occipital nerve block \\ TPI - trigger point injection \\ BA - brush allodynia \\ EM - episodic migraine \\ TM - transformed migraine \\ VAS - visual analog scale \\ NSAIDs - non steroidal anti-inflammatory drugs \\ DNIC - diffuse noxious inhibitory control \\ TNC - trigeminal nucleus caudalis
}




\section{Introduction}

Patients with migraine often have increased skin sensitivity to non-noxious stimuli (cutaneous allodynia). ${ }^{1,2}$ This phenomenon is related to sensitization of central pain neurons receiving convergent input from both intracranial structures and skin. ${ }^{3,4}$ Greater occipital nerve block (GONB) is an effective treatment for migraine pain in a significant

proportion of patients. ${ }^{5}$ It may alleviate head pain by altering the nociceptive input to the trigemino-cervical complex. Its effect on cutaneous allodynia in migraine, however, is currently unknown.

The aim of this study was to evaluate the effect of GONB, with or without trigger point injection (TPI), on dynamical mechanical (brush) allodynia (BA) and on head pain in patients with migraine.

\section{Patients and Methods}

This study was approved by the Institutional Review Board of Jefferson University Hospital. We studied adult (age $>18$ years) patients with IHS-defined episodic migraine (EM) or transformed migraine (TM) as defined by Silberstein and Lipton ${ }^{6}$. All patients had BA and were treated with GONB with or without trigger point injection (TPI). GONB was performed by injecting $2 \mathrm{cc}$ of $2 \%$ lidocaine and $5 \mathrm{mg}$ triamcinolone, $2 \mathrm{~cm}$ lateral to the occipital protuberance. For TPI, $0.5 \mathrm{cc}$ of $2 \%$ lidocaine was injected intramuscularly. Demographic data, migraine history and characteristics of the pain were 
documented. A migraine score was calculated by multiplying the attack frequency (per month) by attack duration (in hours).

An allodynia questionnaire was given to the patients. It consisted of questions about head pain evoked or made worse by various non-noxious stimuli: tilting or bending down the head, coughing, climbing stairs, touching the scalp or face, shaving (for men) or wearing jewelry (for women), wearing tight collars and exposure to wind. An allodynia index was determined by adding the number of positive answers to the above seven questions, with a result ranging from 0 to 7 . Allodynia was also evaluated by repetitively applying a $4 \times 4$ inch gauze pad to various skin areas in the trigeminal (V1, V2, V3) and cervical (C2, C5, C8) dermatomes. The gauze was applied ten times at a rate of $2 / \mathrm{sec}$. Degree of allodynia (the allodynia score) was measured on a $100 \mathrm{~mm}$ visual analog scale (VAS) before treatment and 10 and 20 minutes thereafter. Allodynia scores, as well as pain levels, before and after treatment were compared. The allodynia scores, obtained by examination, were also compared to the allodynia index as obtained per history.

\section{Results}

Nineteen patients (15 females and four males) were studied. Mean age was $43.6 \pm 11.8$ years. Eighteen patients had transformed migraine (TM) and one had episodic migraine (EM). Four underwent GONB alone and 15 had GONB with TPI. Seven patients had an acute IHS-defined migraine attack when treated, with an average time of $29.2 \pm 11.3$ hours between the beginning of the attack and the GONB treatment. Six patients had taken an acute migraine drug during the 24 hour period prior to GONB treatment. The shortest 
interval between acute medication treatment and GONB was 3 hours. Seventeen patients were on migraine-preventive drugs [12 on antidepressants (nortriptyline, amitriptyline, desipramine, venlafaxine, fluoxetine, sertraline), 12 on anticonvulsants (topiramate, valproic acid, oxcarbazepine, gabapentin, levetiracetam), 5 on calcium channel antagonists (verapamil, amlodipine), 3 on NSAIDs (naproxen, celecoxib, rofecoxib), 3 on muscle relaxants (tizanidine, metaxalone) and 3 on antipsychotics (chlorpromazine, quetiapine, ziprasidone)] with an average of 2.82 drugs per patient.

Twenty minutes after treatment, pain was reduced in 17 patients $(89.5 \%)$ and did not change in two $(10.5 \%)$. The average pain score before treatment was 6.53 (on a scale of $0-10)$ and it decreased to 3.47 twenty minutes after treatment $(\mathrm{P}<0.001)$ (Figure 1). In the cervical dermatomes, the average allodynia score was significantly higher at C2 (28.39) compared to both $\mathrm{C} 5(19.00, \mathrm{p}=0.014)$ and $\mathrm{C} 8(16.13, \mathrm{p}=0.002)$ prior to treatment (Figure 2). The average allodynia scores decreased after 20 minutes for all patients. Average allodynia score per site in the trigeminal areas was reduced by $18.69 \mathrm{~mm}$ (from 28.42 before treatment to 9.73 after it) and in the cervical areas it was reduced by $13.74 \mathrm{~mm}$ (from 20.08 to 6.34$)(\mathrm{p}<0.001)$ (Figure 3). Average allodynia was (non-significantly) more severe on the left (26.0) compared with the right (22.5) prior to treatment $(\mathrm{p}=0.594)$. Head pain reduction was (non-significantly) smaller on the left $(2.50$ [from 7.25 to 4.75$])$ compared with the right (3.87 [from 6.50 to 2.63$])(p=0.099)$.

Data analysis on the side of GONB in relation to the side of allodynia reduction showed that GONB reduced allodynia scores by $65.7 \%$ ipsilaterally and by $59.2 \%$ contralaterally. The average allodynia index (per history) for the entire group was 4.16 (on a scale of 0 7). There was a significant positive correlation of between the average allodynia score 
and the allodynia index $(\mathrm{p}=0.014)$ (Figure 4). A positive correlation between the allodynia score and the migraine score did exist, however, that correlation did not reach statistical significance $(\mathrm{p}=0.304)$.

\section{Comments}

GONB, with or without TPI, reduced both head pain and allodynia in this study. The high proportion of patients responding to GONB in our study $(89.5 \%)$ is similar to that found in previous reports. ${ }^{5}$ The effect of GONB on allodynia in our study was similarly high $(100 \%)$

Our results suggest an effect of GONB on sensitized neurons in the trigemino-cervical complex, resulting in a reduction of both head pain and cutaneous allodynia. The mechanism of action of GONB is incompletely understood. It may be related to the anesthetic effects of the drugs injected, or to the physical effect of the injection itself on the nerve via a diffuse noxious inhibitory control (DNIC) mechanism. ${ }^{7,8}$ Since we did not compare the effect of GONB with anesthetic drugs to that of sham injection of the nerve, we can not conclude as to the exact mechanism of action of GONB, nor could we assess the extent of a placebo effect of the procedure.

The results of allodynia testing in our study may have been affected by the preventive medications that the majority of patients were on. However, the exact effect of preventive medications on the occurrence of cutaneous allodynia in migraine is currently unknown. Other limitations of the study are the heterogeneity of the study population and the fact that some patients had TPI in addition to the GONB. In addition, some patients were overusing acute pain medications, which may have affected their response to GONB. 
The effect of GONB on allodynia in the different dermatomes was not uniform. It was significantly higher in the trigeminal area compared with the cervical dermatomes. This suggests, but does not prove, a contiguous spread of GONB effect on sensitized neurons in the trigemino-cervical complex, with neurons in the trigeminal nucleus caudalis (TNC) affected first and to a higher degree than those in the cervical segment of the spinal cord. An alternative explanation is that neuronal sensitization in migraine is a process involving first-, second- and third order neurons sequentially as suggested by Burstein et al. ${ }^{1,2}$ These hypotheses need to be further assessed by electrophysiologic studies, such as single cell recordings from neurons in the TNC, cervical spinal cord and thalamus in animal models. It should be noted that the results of allodynia reduction in the cervical dermatomes need to be interpreted with caution since some patients were given TPI in the same or adjacent areas to the ones tested for allodynia.

In this study we show a significant reduction of allodynia by GONB not only ipsilaterally but also, and to a similar extent, contralateral to the block. This suggests a spread of the GONB effect across the midline, through a multi-synaptic pathway. The anatomy of this putative pathway, however, is currently unknown.

The severity of allodynia may correlate with migraine attack frequency and duration (which is presented in our study as the migraine score) although this correlation did not reach statistical significance. This suggests that allodynia may be a manifestation of longstanding neuronal hyper-excitability which in turn results from repeated frequent migraine attacks. This notion is supported by recent findings of a correlation between the 
duration of disease and the occurrence of cutaneous allodynia in cluster headache patients 9

Analysis of our data separately for head pain in each side showed that pain reduction after GONB was less pronounced on the side where allodynia was more severe (left). This is in accordance with previous reports showing that the development of cutaneous allodynia in migraine is a predictor of poor response to treatment of a migraine attack with triptans ${ }^{10}$. Finally, we found our allodynia questionnaire to be valid and to correlate well with the scores of allodynia per examination.

In summary, in this study we show that GON block, with or without TPI, reduces both pain and allodynia in migraine patients. This pilot study should be followed by a larger controlled trial to further assess the effects of GON block and TPI on headache and allodynia in migraine, and to differentiate between the effects of the two procedures on these parameters. 


\section{REFERENCES}

1. Burstein R, Yarnitsky D, Goor-Aryeh I, Ransil BJ, Bajwa ZH. An association between migraine and cutaneous allodynia. Ann Neurol 2000;47:614-624.

2. Burstein R, Cutrer MF, Yarnitsky D. The development of cutaneous allodynia during a migraine attack clinical evidence for the sequential recruitment of spinal and supraspinal nociceptive neurons in migraine. Brain 2000;123 ( Pt 8):17031709.

3. Burstein R, Yamamura H, Malick A, Strassman AM. Chemical stimulation of the intracranial dura induces enhanced responses to facial stimulation in brainstem trigeminal neurons. J Neurophysiol 1998;79:964-982.

4. Willis WD. Role of neurotransmitters in sensitization of pain responses. Ann NY Acad Sci 2001;933:142-156.

5. Caputi CA, Firetto V. Therapeutic blockade of greater occipital and supraorbital nerves in migraine patients. Headache 1997;37:174-179.

6. Silberstein SD, Lipton RB, Sliwinski M. Classification of daily and near-daily headaches: field trial of revised IHS criteria. Neurology 1996;47:871-875.

7. Witting N, Svensson P, Arendt-Nielsen L, Jensen TS. Differential effect of painful heterotopic stimulation on capsaicin-induced pain and allodynia. Brain Res 1998;801:206-210.

8. Bouhassira D, Danziger N, Attal N, Guirimand F, Atta N. Comparison of the pain suppressive effects of clinical and experimental painful conditioning stimuli. Brain 2003;126:1068-1078.

9. Ashkenazi A, Young WB. Brush allodynia in cluster headache. Headache 2004;44:1010-1012

10. Burstein R, Collins B, Jakubowski M. Defeating migraine pain with triptans: a race against the developing allodynia. Ann Neurol 2004;55:19-26. 
Legends:

Figure 1: Average pain reduction after treatment with GONB \pm TPI

Figure 2: Average allodynia scores per site before treatment in the different areas

Figure 3: Average allodynia scores per site before and after treatment

Figure 4: Correlation between the allodynia index (obtained by a questionnaire) and allodynia scores (obtained by examination) 
Fig. 1

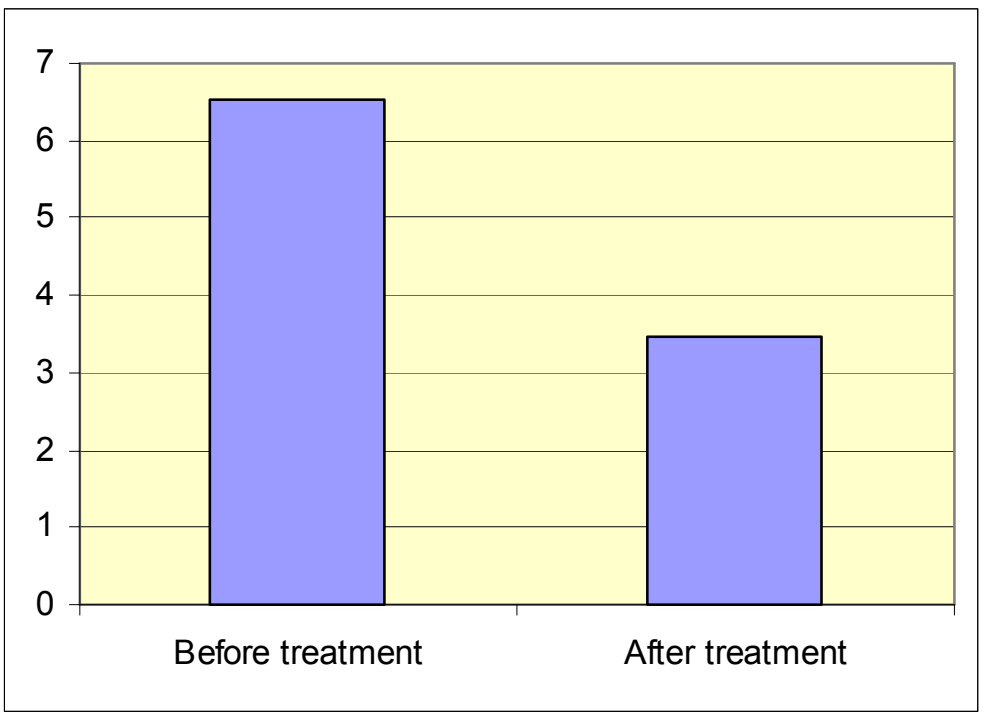

This is the authors' final version prior to publication in Headache: The Journal of Head and Face Pain 45(4):350-354, April 2005. (http://dx.doi.org/ 10.1111/j.1526-4610.2005.05073.x) Copyright C Blackwell Publishing, Inc. 
Fig. 2

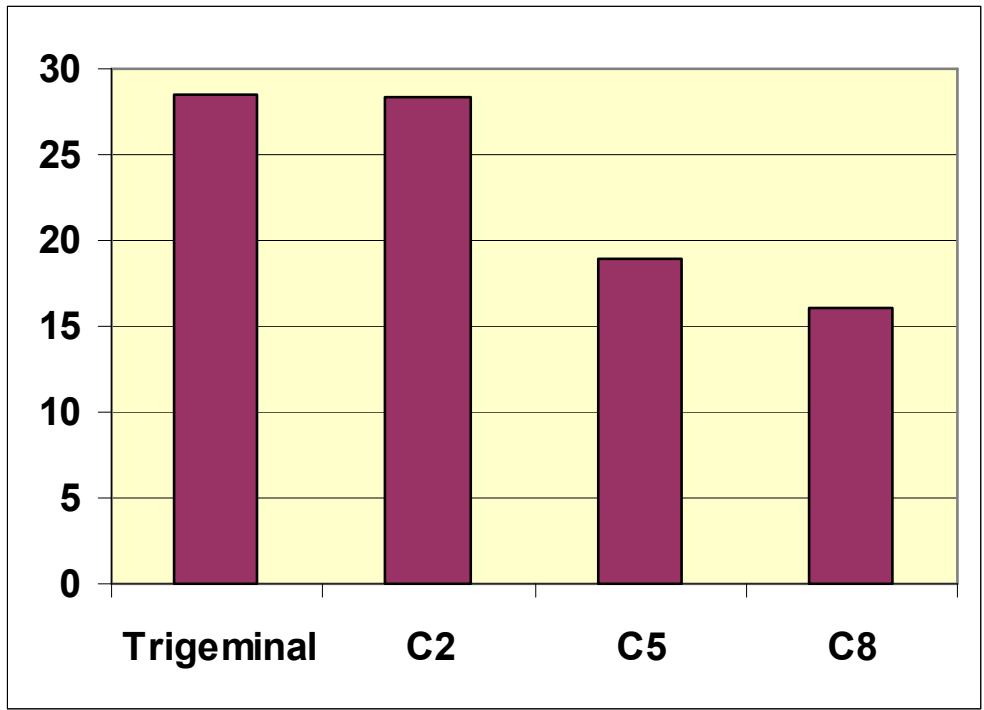

This is the authors' final version prior to publication in Headache: The Journal of Head and Face Pain 45(4):350-354, April 2005. (http://dx.doi.org/ 10.1111/j.1526-4610.2005.05073.x) Copyright (C) Blackwell Publishing, Inc. 
Fig. 3

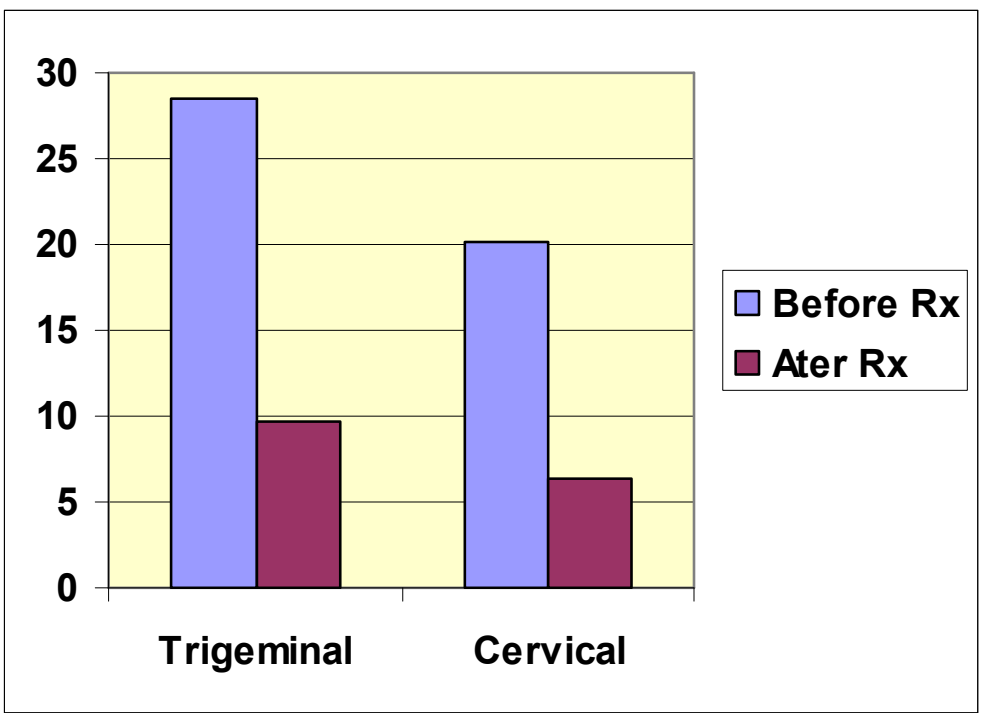

This is the authors' final version prior to publication in Headache: The Journal of Head and Face Pain 45(4):350-354, April 2005. (http://dx.doi.org/ 10.1111/j.1526-4610.2005.05073.x) Copyright $($ C Blackwell Publishing, Inc. 
Fig.4

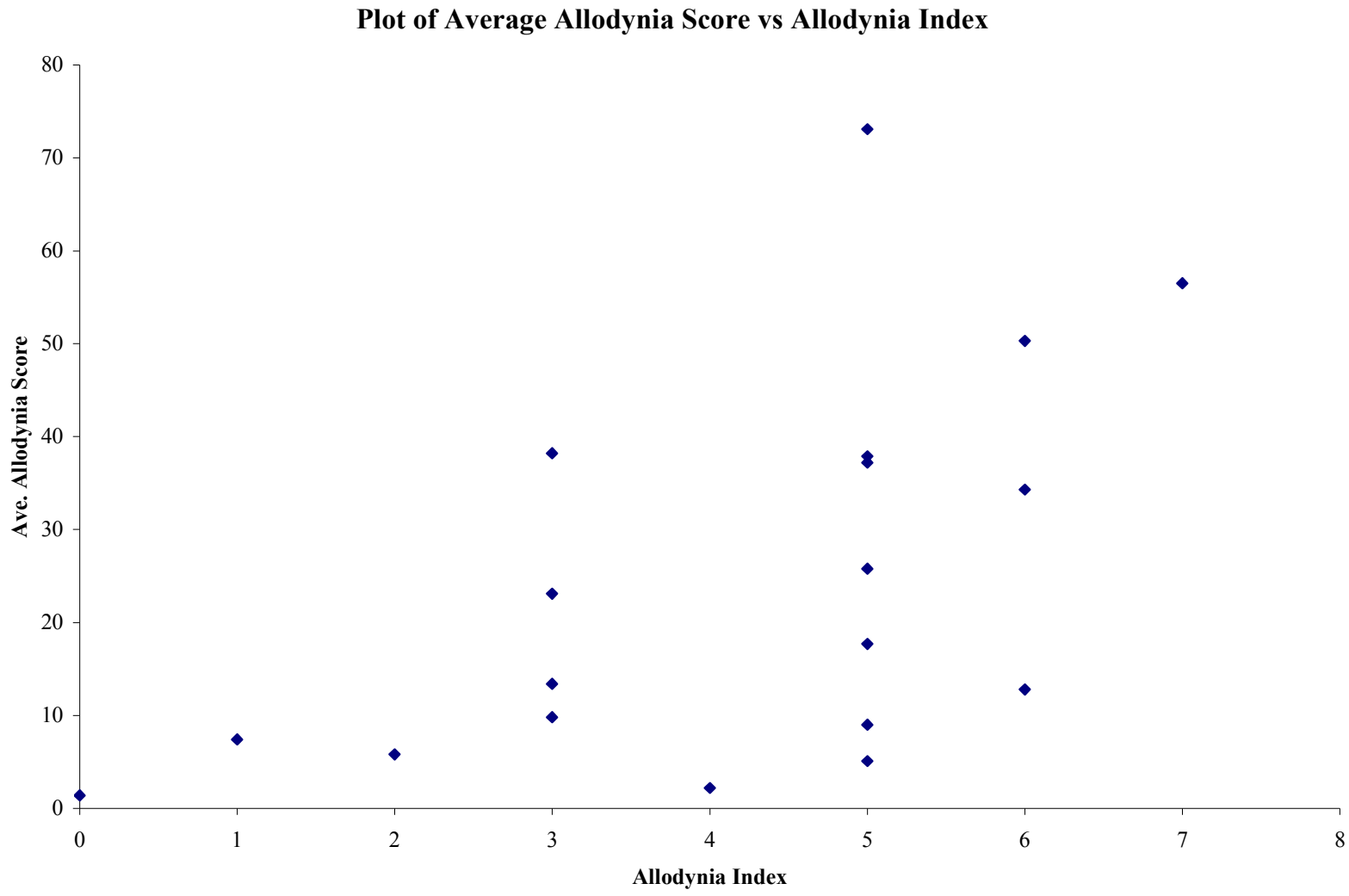

This is the authors' final version prior to publication in Headache: The Journal of Head and Face Pain 45(4):350-354, April 2005. (http://dx.doi.org/ 10.1111/j.1526-4610.2005.05073.x) Copyright C Blackwell Publishing, Inc. 